\title{
Status of Tau Physics
}

\author{
Alan J. Weinstein \\ California Institute of Technology, Pasadena, California 91125, USA
}

\begin{abstract}
We review, very briefly, many of the important results arising in the study of tau lepton production and decay, with emphasis on recent results. We discuss the studies of topological and exclusive branching fractions, inclusive semi-leptonic tau decay spectral functions, extraction of QCD parameters including $\alpha_{S}$ and the strange quark mass, exclusive semileptonic decays and low energy meson dynamics, and rare and forbidden (neutrinoless) decays. Special attention is paid to recent searches for $\mathrm{CP}$ non-conservation in tau pair production and tau decay.
\end{abstract}

\section{INTRODUCTION}

The tau is considered to be a heavy flavor, hence its token appearance at this conference. That it is "heavy" is borne out by the fact that the tau has many decay modes, and thus a rich phenomenology. Its mass is large compared to the scale of hadronization: $m_{\tau} \gg \Lambda_{Q C D}$. Thus, decays of the tau can be used to probe QCD in the perturbative regime, as discussed in the section on inclusive semileptonic decays.

Most importantly, the tau appears to be a pointlike fundamental fermion, and its electroweak couplings can be measured with precision that, in some cases, can exceed that of the lighter leptons. It is thus a useful tool for probing some of the least well understood aspects of particle physics: the dynamics of light mesons at low energies, and physics beyond the Standard Model (SM).

Tau physics is a large field. Since this is a status report, I endeavor to cover many of the important results, but necessarily, in a very brief manner. Special attention is paid to recent searches for $\mathrm{CP}$ violation in tau production and decay. Explicitly omitted from this report are discussions of tau neutrino properties, leptonic decays, tests of lepton universality, Michel parameters, and neutral current couplings.

\section{Progress since Heavy Flavors 8}

Progress in tau physics has tapered off in the last couple of years. The LEP program is over, and final results in for $Z^{0} \rightarrow \tau^{+} \tau^{-}$from LEP I and $W^{ \pm} \rightarrow \tau^{ \pm} \nu_{\tau}$ from LEP II (all results are nicely consistent with the SM). There were many beautiful results presented at the TAU2000 workshop in Victoria, Canada [1].

There have been recent results from LEP on Michel parameters from DELPHI [2] and ALEPH [3], on precision topological branching fractions from L3 [4] and DELPHI [5], as well as final results from all LEP collaborations on tau polarization at the $Z^{0}$ peak.

CP618, Heavy Flavor Physics: Ninth International Symposium, edited by A. Ryd and F. C. Porter () 2002 American Institute of Physics 0-7354-0064-4/02/\$19.00

447 
The results on hadronic contributions to the muon anomalous magnetic moment, based on ALEPH data from tau decays [6], have fueled the debate over whether the recent precision measurement of $a_{\mu}$ from BNL are in conflict with the SM.

CLEO has published results on structure in hadronic decays containing charged kaons [7], the branching fraction for the rare decay to $6 \pi \nu$ [8], and two new papers on searches for $\mathrm{CP}$ violation in tau decays $[9,10]$.

Belle has presented first results on tau physics at this summer's conferences, including searches for neutrinoless tau decays [11, 12], for CP violation in tau decays [13], and for a CP-violating EDM in tau pair production [14].

\section{TOPOLOGICAL AND EXCLUSIVE BRANCHING FRACTIONS}

There are new precision measurements on tau decay topological (charged particle multiplicity) branching fractions from L3 [4] and DELPHI [5], with errors of the same order as the PDG2000 world averages [15]. In these measurements, charged tracks from $K_{S}^{0}$ decays, Dalitz decays, and photon conversions do not contribute to the charged particle multiplicity. The results from $\mathrm{L} 3$ are:

$$
\begin{aligned}
\mathcal{B}(1-\text { prong }) & =(85.27 \pm 0.11 \pm 0.07) \% \\
\mathcal{B}(3-\text { prong }) & =(14.56 \pm 0.11 \pm 0.08) \% \\
\mathcal{B}(5-\text { prong }) & =(0.17 \pm 0.02 \pm 0.03) \%
\end{aligned}
$$

All conventional exclusive final states are reasonably well measured. The final states used in the PDG fit include:

$$
\begin{gathered}
e^{-} v v_{\tau}, \mu^{-} v v_{\tau}, \pi^{-} v_{\tau}, \pi^{-} \pi^{0} v_{\tau}, \pi^{-} 2 \pi^{0} v_{\tau}, \pi^{-} 3 \pi^{0} v_{\tau}, \pi^{-} 4 \pi^{0} v_{\tau}, \\
\pi^{-} \pi^{+} \pi^{-} v_{\tau}, \pi^{-} \pi^{+} \pi^{-} \pi^{0} v_{\tau},(5 \pi)^{-} v_{\tau},(6 \pi)^{-} v_{\tau}, \\
K^{-} v_{\tau}, K^{-} \pi^{0} v_{\tau}, \pi^{-} \bar{K}^{0} v_{\tau},(K \pi \pi)^{-} v_{\tau},(K 3 \pi)^{-} v_{\tau}, K^{-} \bar{K}^{0} v_{\tau} \\
(K K \pi)^{-} v_{\tau},(K K 2 \pi)^{-} v_{\tau}, \eta \pi^{-} \pi^{0} v_{\tau}, \eta K^{-} v_{\tau} .
\end{gathered}
$$

Exclusive final states saturate these topological branching fractions nicely, and sum to one. The constrained PDG 2000 fit to the branching fractions agree well with the averages of the direct measurements (in contrast to previous years).

\section{INCLUSIVE SEMI-LEPTONIC DECAYS}

The semi-leptonic decays of the tau to $v_{\tau}$ plus hadrons provides a clean source of hadrons with invariant mass below $m_{\tau}$ for the study of low-energy meson dynamics. The hadronic system in $\tau \rightarrow \nu_{\tau} X$ can be studied in terms of its exclusive final state (e.g., $\pi, 2 \pi, 3 \pi, K \pi$, etc.), or inclusively, broken down by invariant mass $s=m_{X}^{2}$, strangeness, and whether the final state proceeds via the vector $V$ or axial-vector $A$ part of the charged weak current. 
Inclusive studies can be cast in terms of the production-independent vector and axialvector spectral functions $\mathrm{v}^{(s)}$ and $a^{(s)}$, where the superscript denotes whether the final state has strangeness or not. One can extract these spectral functions directly from the data; $e . g$., the $\pi \pi$ contribution to $\mathrm{v}(s)$ is obtained from

$$
v^{\pi \pi}(s) \sim \underbrace{\frac{B_{\pi \pi^{0}}}{B_{e}}}_{\text {Branching ratios Mass spectrum }} \underbrace{\frac{1}{N_{\pi \pi^{0}}} \frac{d N_{\pi \pi^{0}}}{d s}}_{\text {Kinematic factor }} \underbrace{\frac{m_{\tau}^{2}}{\left(1-s / m_{\tau}^{2}\right)^{2}\left(1+2 s / m_{\tau}^{2}\right)}}_{\text {Mas/ }} .
$$

The Conserved Vector Current (CVC) hypothesis relates the vector part of the hadronic spectral function measured in weak decays to the same quantity measured in electromagnetic processes. An isospin rotation connects the charged weak hadronic spectral function to the isospin-1 part of the neutral hadronic spectral function. Thus, the vector spectral function measured in tau decays, $\tau^{-} \rightarrow \nu_{\tau} W^{-}, W^{-} \rightarrow$ hadrons, can be compared with that measured in, e.g., $e^{+} e^{-} \rightarrow \gamma^{*}, \gamma^{*} \rightarrow$ hadrons:

$$
\sigma\left(e^{+} e^{-} \rightarrow \pi^{+} \pi^{-}\right)=\frac{4 \pi \alpha^{2}}{s} \mathrm{v}_{I=1}^{\pi \pi}
$$

as a test of CVC and isospin symmetry. In this example, the $I=0$ part of the $e^{+} e^{-}$ cross section $\left(e^{+} e^{-} \rightarrow \omega \rightarrow \pi^{+} \pi^{-}\right)$must be subtracted out. A comparison of the $\mathrm{v}^{\pi \pi}(s)$ measured in these two ways [16] is shown in Fig. 1, as a test of CVC.

Assuming CVC and isospin symmetry, the vector spectral functions measured in tau decays can be used to improve the estimate of the contribution of hadronic vacuum polarization to the running QED coupling constant $\alpha_{Q E D}\left(m_{Z}^{2}\right)$ and the muon anomalous magnetic moment $a_{\mu}^{\text {had }}$. The values of these quantities so obtained [6] constitute some of the most accurate estimates of these quantities.

The total ratio of hadrons to leptons in tau decays can be extracted from the world average [15] leptonic branching fractions alone:

$$
R_{\tau} \equiv \frac{\Gamma\left(\tau^{-} \rightarrow v_{\tau} \text { hadrons }\right)}{\Gamma\left(\tau^{-} \rightarrow v_{\tau} e^{-} \bar{v}_{e}\right)}=\frac{1-\mathcal{B}_{e}-\mathcal{B}_{\mu}}{\mathcal{B}_{e}}=3.642 \pm 0.019
$$

The dependence of $R_{\tau}\left(s_{0}\right)$ on the maximum hadronic invariant mass can be extracted from the inclusive vector and axial-vector spectral functions. These have been measured/estimated in tau decays by ALEPH [17] and OPAL [18], with good agreement. The results from ALEPH are shown in Fig. 2.

The fact that these spectral functions, and their integrals, flatten out as $s_{0}$ approaches $m_{\tau}^{2}$ is qualitative evidence that $m_{\tau}^{2}$ is "QCD asymptopia", so that QCD perturbative methods and the Operator Product Expansion can be applied to understand the tau data at the tau mass scale. In this sense, we can regard the tau as a "heavy flavor".

The inclusive spectral functions can be used to extract QCD perturbative parameters $\left(\alpha_{S}\left(m_{\tau}^{2}\right)\right)$, quark masses $\left(m_{q}\left(m_{\tau}^{2}\right)\right)$, and non-perturbative condensates [19]: 


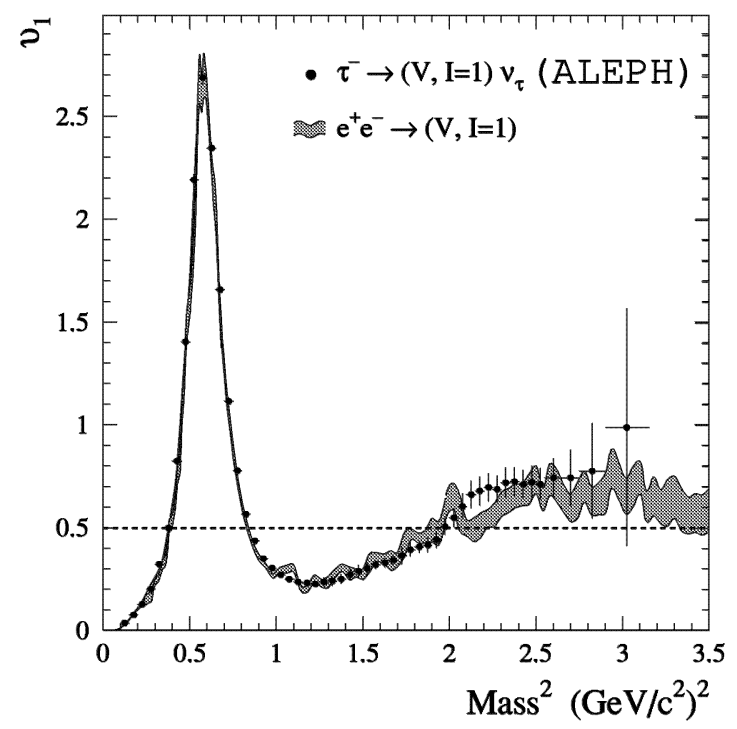

FIGURE 1. Total hadronic vector current spectral function from tau decays measured by ALEPH (data points), and the corresponding distribution calculated from $e^{+} e^{-}$isovector states using isospin symmetry (shaded band). The dashed line corresponds to the naive isovector quark-parton prediction. Taken from [16] with permission, (c) Springer-Verlag 1997.
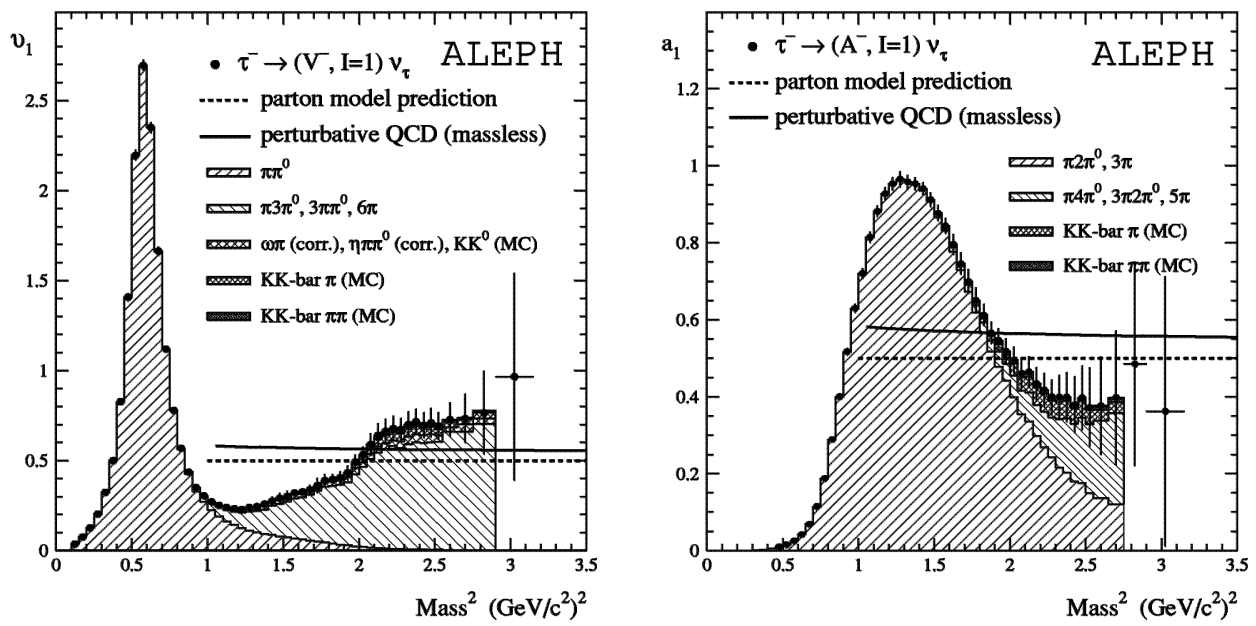

FIGURE 2. The total vector (left) and axial-vector (right) spectral functions from tau decays measured by ALEPH (data points), broken down by exclusive final state contributions (some of which are estimated from Monte Carlo simulations). The lines show the predictions from the naive parton model and from massless QCD using $\alpha_{S}\left(M_{Z}^{2}\right)=0.120$. Taken from [17] with permission, @ Springer-Verlag 1998. 


$$
R_{\tau V, A}=\frac{3}{2}\left|V_{u d}\right|^{2} \underbrace{S_{E W}}_{\text {known }}(1+\underbrace{\delta^{(0)}\left(\alpha_{S}\left(m_{\tau}^{2}\right)\right)}_{\text {perturb }}+\underbrace{\delta_{E W}^{\prime}}_{\text {known }}+\underbrace{\delta_{V, A}^{(2, m)}\left(m_{u d}^{2}\right)}_{\text {mass-dependence }}+\underbrace{\sum_{D=4,6, \cdots} \delta_{u d, V, A}^{(D)}}_{\text {non-perturb }}) .
$$

The dependence of the perturbative correction $\delta^{(0)}$ on $\alpha_{S}\left(m_{\tau}^{2}\right)$ is calculated up to fourth order in fixed-order perturbation theory [19]. The quark mass dependent nonperturbative correction $\delta_{V, A}^{(2, m)}\left(m_{u d}^{2}\right)$ has been calculated [20] up to next-to-leading order in $\alpha_{S}$.

From fits to $R_{\tau}$ and the moments of $R_{\tau}\left(s_{0}\right)$, ALEPH extracts [17] $\alpha_{S}\left(m_{\tau}^{2}\right)$, and runs the value up to the $Z^{0}$ mass scale:

$$
\alpha_{S}\left(m_{\tau}^{2}\right)=0.334 \pm 0.022 \quad \Longrightarrow \quad \alpha_{S}\left(m_{Z}^{2}\right)=0.1202 \pm 0.0027 .
$$

This agrees well with other measures of $\alpha_{S}\left(m_{Z}^{2}\right)$ [21], and constitutes one of the most precise measurements of this quantity.

\section{Strange quark mass}

Considering the decays of the tau to final states containing net strangeness $\left(\mathrm{K}^{-}\right.$, $(K \pi)^{-},(K 2 \pi)^{-}$, etc.), the contribution to $R_{\tau, S}$ depends on $\delta_{V, A}^{(2, m)}\left(m_{s}^{2}\right)$, which in turn depends on the strange quark mass evaluated at the tau mass scale, $m_{s}\left(m_{\tau}^{2}\right)$. The perturbative expansion exhibits bad behavior, so care must be taken to extract a meaningful estimate of the quark mass [22]. Using data from ALEPH, CLEO, and world-average branching fractions for $\tau^{-} \rightarrow v_{\tau}(K n \pi)^{-}$decays, Ref. [22] obtains

$$
R_{\tau, S}=0.1630 \pm 0.0057 \quad \text { and } m_{s}\left(m_{\tau}^{2}\right)=120_{-26}^{+21} \mathrm{MeV}
$$

\section{EXCLUSIVE SEMILEPTONIC DECAYS}

Semi-leptonic tau decays occur at relatively low momentum transfer. Although the inclusive sum of all the final state modes approaches a limit where QCD perturbative expansions can be applied, the study of exclusive final states relies instead on models of low-energy meson dynamics, resonance dominance, and to some extent, chiral perturbation theory. ARGUS, CLEO, and the LEP experiments have performed many detailed studies of the resonant structure of exclusive final states, and have extracted resonance parameters, sub-resonances, and couplings between vector, axial-vector, scalar and tensor resonances. These measurements have been used to test CVC, test the spin structure in tau decay, look for second-class (isospin-violating) currents, etc.

There are many resonances that contribute to the structure of of hadronic final states in tau decays. The $\pi^{-} \pi^{0}$ final state proceeds through the $\rho(770)$ and its radial excita- 
tions. The $3 \pi$ final state proceeds through the $a_{1}(1260)$, which decays to $\rho \pi$ and $\rho^{\prime} \pi$ via both s-wave and $\mathrm{d}$-wave amplitudes, and to isoscalars $\sigma(400-1200) \pi, f_{2}(1270) \pi$ and $f_{0}(1370) \pi$ via p-wave amplitudes. There have been (unsuccessful) searches for a contribution from $\pi^{\prime}(1300) \rightarrow \rho \pi$ or $\sigma \pi$. The $4 \pi$ final state proceeds through the $\rho^{\prime}$, decaying to $\omega \pi, a_{1} \pi$, and $\rho \pi \pi$. There have been (unsuccessful) searches for a contribution from $b_{1}(1235) \rightarrow \omega \pi$, which would be an isospin-violating second-class current [23]. The $\eta \pi^{-}$final state $[24,25]$ is forbidden by isospin (it is a second-class current), and the $\eta \pi^{-} \pi^{0}$ final state proceeds via the Wess-Zumino chiral anomaly [25]. The $5 \pi$ and $6 \pi$ final state resonant substructure is so complex, and the data samples so limited, that they have yet to be explored with any precision [26].

Final states containing kaons have also been studied [27]. The $K \pi$ final state is dominated by the $K^{*}(892)$, although redial excitations (the $K^{*}(1410)$ ) may also contribute. The $K \pi \pi$ final state proceeds through the $K_{1}(1270)$ and, to a lesser extent, the $K_{1}(1400)$, both of which decay to $K^{*} \pi$ and $K \rho$. The $K_{1}(1270)$ and $K_{1}(1400)$ are mixtures of the $K_{1 a}$ (in the $J^{P C}=1^{++}$octet) and the $K_{1 b}$ (in the $J^{P C}=1^{+-}$octet); the latter couples to the weak charged current only through $S U(3)_{f}$ violation. The $K K \pi$ final states can proceed through the axial-vector $a_{1}$. The $\eta K^{-}$final state has been observed [24]; it is an $S U(3)_{f}$ violating transition.

This brief status report cannot do justice to this rich subject; refer to Ref. [28] for more details.

\section{RARE AND FORBIDDEN DECAYS}

There are a variety of rare but allowed decays that have been seen or searched for in tau decays. Some interesting recent measurements include:

$$
\begin{array}{lll}
\mathcal{B}\left(\tau \rightarrow \eta 3 \pi v_{\tau}\right) & =(4.8 \pm 1.1) \times 10^{-4} & \text { (CLEO 98 [29]) } \\
\mathcal{B}\left(\tau \rightarrow \eta K v_{\tau}\right) & =(2.7 \pm 0.6) \times 10^{-4} & \text { (CLEO\&ALEPH [24]) } \\
\mathcal{B}\left(\tau \rightarrow \eta K \pi v_{\tau}\right) & =(5.0 \pm 1.2) \times 10^{-4} & \text { (CLEO 98 [24]) } \\
\mathcal{B}\left(\tau \rightarrow e^{-} e^{+} e^{-} v v_{\tau}\right) & =(2.8 \pm 1.5) \times 10^{-5} & \text { (CLEO 98 [30]) } \\
\mathcal{B}\left(\tau \rightarrow \pi^{-} K^{0} \bar{K}^{0} \pi^{0} v_{\tau}\right) & =(3.1 \pm 2.3) \times 10^{-4} & \text { (ALEPH 99 [22]) } \\
\mathcal{B}\left(\tau \rightarrow \pi^{-} K^{+} K^{-} \pi^{0} v_{\tau}\right) & =(4.0 \pm 1.6) \times 10^{-4} & \text { (CLEO\&ALEPH [27]) } \\
\mathcal{B}\left(\tau \rightarrow K^{-} K^{+} K^{-} v_{\tau}\right) & <1.9 \times 10^{-4} & \text { (ALEPH 98 [31]) } \\
\mathcal{B}\left(\tau \rightarrow 7-\text { prong } \nu_{\tau}\right) & <2.4 \times 10^{-6} & \text { (CLEO 97 [32]) } \\
\mathcal{B}\left(\tau \rightarrow \eta \pi v_{\tau}\right) & <1.4 \times 10^{-4} & \text { (CLEO 96 [24]) } \\
\mathcal{B}\left(\tau \rightarrow(\omega \pi)_{1++} v_{\tau}\right) & <1.3 \times 10^{-3} & \text { (CLEO 99 [23]) }
\end{array}
$$

The last two upper limits correspond to isospin-violating second-class currents. 


\section{Limits on neutrinoless decays}

There are several classes of decay modes that are forbidden in the SM, usually because they produce no $v_{\tau}$ in the final state. These include:

- Lepton Flavor Violating (LFV) decays like $\tau^{-} \rightarrow \mu^{-} \gamma, \tau^{-} \rightarrow e^{-} e^{+} e^{-}$

- Lepton number violating (LV) decays like $\tau^{-} \rightarrow \mu^{+} \pi^{-} \pi^{-}, \mu^{+} e^{-} e^{-}$

- B-L conserving modes like $\tau^{-} \rightarrow p^{-} \gamma$

- Exotic modes like $\tau^{-} \rightarrow \pi^{-} v_{\text {heavy }}, e^{-} G^{0}$, where $v_{\text {heavy }}$ is a neutral heavy fermion and $G^{0}$ is a neutral heavy boson

- Angular momentum violating modes like $\tau^{-} \rightarrow \pi^{-} \pi^{0}, \pi^{-} \gamma$

CLEO [33] has set limits on a long list of such decays, typically resulting in branching fraction upper limits of a few $\times 10^{-6}$ at $95 \%$ C.L. The angular momentum violating modes, and the $e^{-} G^{0}, \mu^{-} G^{0}$ modes were studied by ARGUS [34]. Belle has recently searched and found no evidence for the decays to $e^{-} K^{0}$ and $\mu^{-} K^{0}$, with branching fraction upper limits of $1.8 \times 10^{-6}$ at $95 \%$ C.L. for both modes [12].

Many extensions of the SM predict that the decay $\tau^{-} \rightarrow \mu^{-} \gamma$ should proceed, and some SUSY and LR-symmetric models predict an enhancement of this decay rate relative to $\mu^{-} \rightarrow e^{-} \gamma$ of $10^{5}-10^{6}$. Recent searches for these modes give:

$$
\begin{aligned}
& \mathcal{B}\left(\mu^{-} \rightarrow e^{-} \gamma\right)<1.2 \times 10^{-11} \quad \text { (MEGA '99 [35]) } \\
& \mathcal{B}\left(\tau^{-} \rightarrow \mu^{-} \gamma\right)<1.1 \times 10^{-6} \quad \text { (CLEO '00 [36]) } \\
& \mathcal{B}\left(\tau^{-} \rightarrow \mu^{-} \gamma\right)<1.0 \times 10^{-6} \quad \text { (Belle '01 [11]) }
\end{aligned}
$$

\section{CP NON-CONSERVATION IN TAU PHYSICS}

In the SM, there is no mechanism for CP violation in the lepton sector. If neutrinos mix, then the analog of the CKM matrix for neutrinos can produce non-zero but immeasurably small CP-violating effects. The search for $\mathrm{CP}$ violation in tau physics is a search for physics beyond the SM.

\section{CP non-conservation in $e^{+} e^{-} \rightarrow \gamma^{*} / Z^{0 *} \rightarrow \tau^{+} \tau^{-}$}

A simple extension to the SM description of tau pair production in $e^{+} e^{-}$collisions is to add tensor couplings, producing anomalous dipole couplings. For example, well below the $Z^{0}$ pole, the QED Lagrangian can be written as [37]:

$$
\begin{gathered}
\mathcal{L}=\mathcal{L}_{S M}+\mathcal{L}_{E}+\mathcal{L}_{M} ; \quad \mathcal{L}_{S M}=e F_{1}\left(q^{2}\right) \bar{\psi} \gamma^{\mu} \psi A_{\mu} ; \\
\mathcal{L}_{E}=\frac{1}{2} \frac{e F_{2}\left(q^{2}\right)}{2 m_{\tau}} \bar{\psi} \sigma^{\mu v} \psi F_{\mu \nu} ; \quad \mathcal{L}_{M}=\frac{-i}{2} \frac{e F_{3}\left(q^{2}\right)}{2 m_{\tau}} \bar{\psi} \sigma^{\mu v} \gamma_{5} \psi F_{\mu \nu},
\end{gathered}
$$


where the anomalous magnetic and electric dipole moments are given by

$$
a_{\tau} \equiv F_{2}\left(q^{2}=0\right) ; \quad d_{\tau} \equiv \frac{e F_{3}\left(q^{2}=0\right)}{2 m_{\tau}} .
$$

Analogous forms which couple the leptons to the $Z^{0}$ permit the definition of the weak anomalous magnetic and electric dipole moments; there is no general connection between the electromagnetic and weak dipole moments. The electric and weak electric dipole moments (EDMs) are CP-violating. The small CP violation due to the CKM phase in the quark sector produces negligibly small values for these dipole moments in the SM.

Anomalous dipole moments due to beyond-SM physics can have a small(2nd order) effect on the total cross section $\sigma$ and the differential cross section $d \sigma / d \cos \theta$. The effect on the tau spins is first order in these dipole moments; thus, the most sensitive searches for measurable dipole moments come from studies in which the tau spins are inferred from their decays.

The $\tau \tau Z^{0}$ coupling is studied at LEP-I and SLD, using a set of optimal CP-odd tensor observables [38], and limits have been placed on anomalous weak dipole moments $\operatorname{Re}\left(a_{\tau}^{W}\right), \operatorname{Im}\left(a_{\tau}^{W}\right), \operatorname{Re}\left(d_{\tau}^{W}\right), \operatorname{Im}\left(d_{\tau}^{W}\right)$. The LEP limits on the CP-violating weak electric dipole moment are [39] (at 95\% C.L.):

$$
\begin{aligned}
\left|R e\left(d_{\tau}^{W}\right)\right| & <3.0 \times 10^{-18} e \cdot \mathrm{cm} \\
\left|\operatorname{Im}\left(d_{\tau}^{W}\right)\right| & <9.2 \times 10^{-18} e \cdot \mathrm{e} \cdot \mathrm{cm} \\
\left|\left(d_{\tau}^{W}\right)\right| & <9.4 \times 10^{-18} e \cdot \mathrm{cm}
\end{aligned}
$$

The optimal observables at the $Z^{0}$ exploit the parity violating net longitudinal spin asymmetry of the produced taus. The optimal observables are different well below the $Z^{0}$, where there is no net longitudinal spin asymmetry, but there exist non-zero transverse spin correlations between the two taus (at the high LEP/SLD energies, these transverse spin correlations are extremely small). These spin correlations are illustrated in Fig. 3. The optimal observables at, e.g., $E_{c m} \approx 10 \mathrm{GeV}$ exploit the transverse spin asymmetry due to a CP-violating electric dipole moment $d_{\tau}^{\gamma}$.

ARGUS has searched for an anomalous EDM using 277K tau pairs produced near $E_{c m} \approx 10.6 \mathrm{GeV}$ [40]. They estimate the tau spin polarization on both sides of the event using "polarimeter vectors" constructed from $\tau \rightarrow e, \mu, \rho$ decays. They construct optimal observables, and find expectation values for these that are consistent with zero (no $\mathrm{CP}$ violation). From this, they extract limits on the tau EDM of $\left|\operatorname{Re}\left(d_{\tau}\right)\right|<4.6 \times 10^{-16} e$ $\mathrm{cm},\left|\operatorname{Im}\left(d_{\tau}\right)\right|<1.8 \times 10^{-16} e \mathrm{~cm}$, both at $95 \%$ C.L. Belle has searched for an anomalous EDM using 9.4 million tau pairs [14], and establishes limits of $\left|\operatorname{Re}\left(d_{\tau}\right)\right|<6.7 \times 10^{-17} e$ $\mathrm{cm},\left|\operatorname{Im}\left(d_{\tau}\right)\right|<2.2 \times 10^{-17} e \mathrm{~cm}$, both at 95\% C.L. These results can be compared with analogous limits for the electron and muon, $\left|d_{e}\right|<4 \times 10^{-27} e \mathrm{~cm}$, and $\left|d_{\mu}\right|<9 \times 10^{-19}$ $e \mathrm{~cm}$.

An anomalous magnetic moment for the tau would lead to enhancement of radiated photons in the reaction $e^{+} e^{-} \rightarrow \gamma^{*} / Z^{0} \rightarrow \tau^{+} \tau^{-} \gamma$. L3 has searched for and found no evidence of anomalous photon production at LEP, and sets the limits [41] $-0.052<$ $\operatorname{Re}\left(a_{\tau}\right)<0.058$ and $\left|\operatorname{Re}\left(d_{\tau}\right)\right|<3.1 \times 10^{-16} e \mathrm{~cm}$, both at 95\% C.L. In Ref. [42], data on 


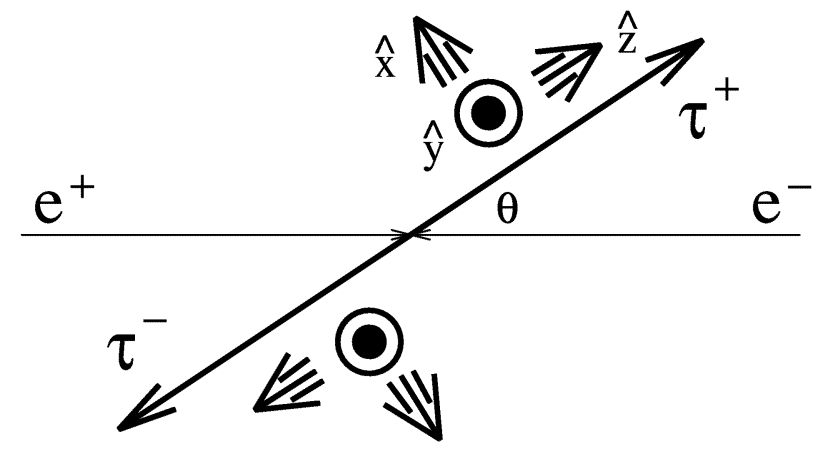

FIGURE 3. Illustration of axes along which tau spins produced in $e^{+} e^{-}$collisions are correlated. The longitudinal ( $\hat{z})$ spin polarization has a net non-zero value at the peak of the $Z^{0}$, but the transverse $(\hat{x}, \hat{y})$ spin polarizations are small. There is no net spin polarization well below the $Z^{0}$, but there are strong spin correlations along all three axes.

the $e^{+} e^{-} \rightarrow \tau^{+} \tau^{-}$cross sections measured at and above the $Z^{0}$ peak, the tau polarization and polarization asymmetry measured at the $Z^{0}$ peak, and the $W \rightarrow \tau \nu$ branching fraction measured at LEPII and the Tevatron, are combined to yield limits on the anomalous magnetic moments of $-0.007<\operatorname{Re}\left(a_{\tau}\right)<0.005$ and $-0.0024<\operatorname{Re}\left(a_{\tau}^{W}\right)<0.0025$, both at the $2 \sigma$ level.

\section{CP non-conservation in tau decay}

One can observe $\mathrm{CP}$ violation in tau decay if it proceeds via two interfering amplitudes (with different angular distributions) and complex relative phases. For example, a charged Higgs could couple to the tau and then to a scalar hadronic system such as a scalar $a_{0}^{-}(980) \rightarrow \pi^{-} \pi^{0}$, as illustrated in Fig. 4. If the $A_{W}$ and $A_{H}$ amplitudes have a relative complex phase, the interference term is $\mathrm{CP}$-odd.

The CP-violating effect is proportional to isospin violation proportional to $\left(m_{u}-m_{d}\right)$ in the decay $\tau \rightarrow \pi^{-} \pi^{0} v_{\tau}$, but the high rate could make such small CP-violating effects observable. In the decay $\tau \rightarrow(K \pi)^{-} v_{\tau}$, the effect requires $\mathrm{SU}(3)$-violation proportional to $\left(m_{u}-m_{s}\right)$, but the rate is much smaller due to Cabibbo suppression.

Both Belle and CLEO have searched for CP violation in tau decays. Belle, using 2.1 million produced tau pairs, looks at single $\tau \rightarrow \pi^{-} \pi^{0} v_{\tau}$ decays. They search for an asymmetry in a CP-odd angular observable, different for $\tau^{+}$and $\tau^{-}$. They see no effect, and set a limit [13] on the asymmetry.

CLEO, using 12.2 million produced tau pairs, searches for CP violation in tau decays to both $\pi^{-} \pi^{0} v_{\tau}$ [9] and $\pi^{-} K_{S}^{0} v_{\tau}$ [10]. In the first analysis, both taus are required to decay to $\pi^{ \pm} \pi^{0}$. All dynamical information in the event is used to construct an optimal observable sensitive to CP-violating asymmetries in the spin correlations between the two decays. No asymmetry in the optimal observable is observed. This is used to set a limit on a model-dependent dimensionless coupling constant $\operatorname{Im}(\Lambda)$ which measures the strength of a Higgs-like coupling relative to the $W$ coupling. A limit of $-0.046<$ 


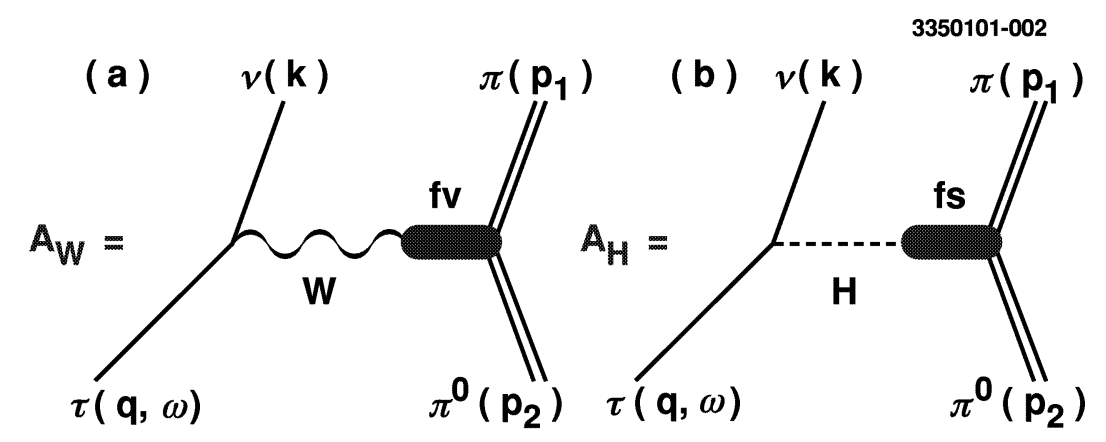

FIGURE 4. Amplitudes for (a) standard $W$ exchange, and (b) scalar (e.g., charged Higgs) exchange, mediating the decay $\tau \rightarrow \pi^{-} \pi^{0} v_{\tau}$. The vector form factor $f_{V}$ is dominated by the rho meson propagator, while the scalar form factor $f_{S}$ might be dominated by the scalar $a_{0}^{-}(980)$. The second diagram could have a weak phase which flips sign under CP.

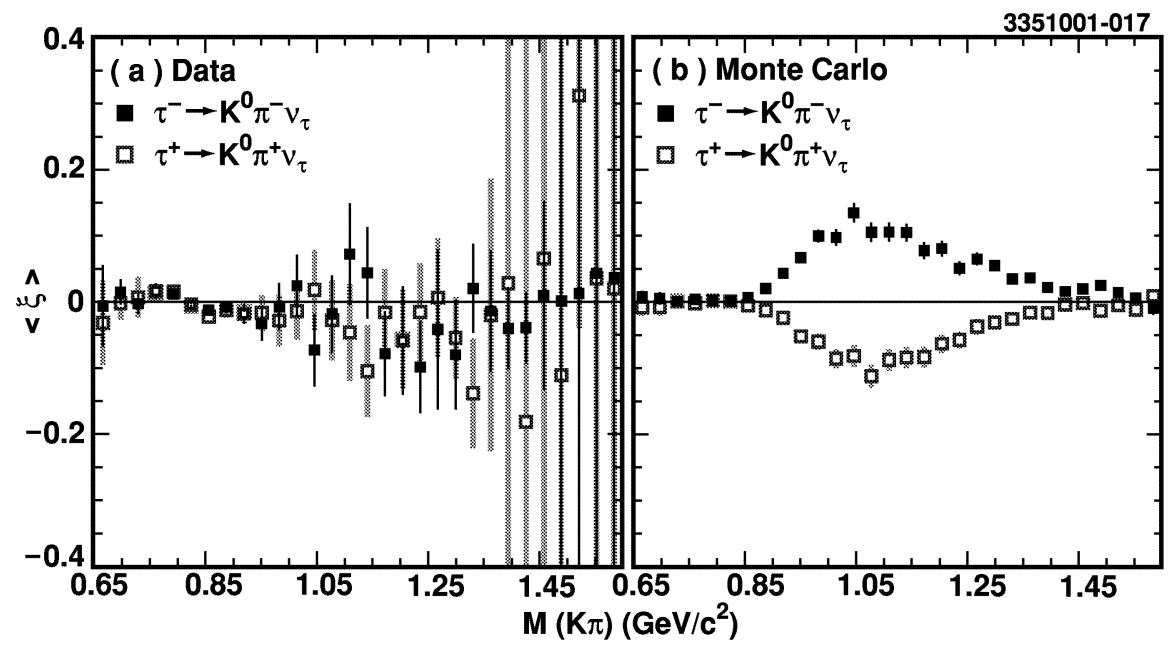

FIGURE 5. Average value of the CP-odd observable $\langle\xi\rangle$ in $\tau \rightarrow \pi^{-} K_{S}^{0} v_{\tau}$ decays as a function of the $\left(\pi^{-} K_{S}^{0}\right)$ invariant mass, for (a) CLEO data and (b) Monte Carlo with maximum CP violation, $\operatorname{Im}(\Lambda)=1$. From [10].

$\operatorname{Im}(\Lambda)<0.022$ at $90 \%$ C.L. is obtained.

In the second analysis, one tau decay to $\pi^{ \pm} K_{S}^{0} v_{\tau}$ is reconstructed; the other tau is used only to tag the event. A CP-odd optimal observable sensitive to the (modeldependent) interference between a vector $K^{*}$ and a scalar $K^{* 0}(1430)$ is constructed. No asymmetry in this observable is seen for either $\tau^{+}$or $\tau^{-}$decays, at any value of $m(K \pi)$ (Fig. 5), and the result is again expressed as a limit on a dimensionless coupling constant $\operatorname{Im}(\Lambda)$ which measures the strength of a Higgs-like coupling relative to the $W$ coupling: $-0.174<\operatorname{Im}(\Lambda)<0.049$ at $90 \%$ C.L. 


\section{FUTURE OF TAU PHYSICS, AND CONCLUSIONS}

The B-Factories (BaBar, Belle) will have produced tau pair samples approaching $10^{8}$ events. This will enable them to push harder on rare decays, make high statistics measurements using modes with kaons, and pursue higher precision on many different topics, such as the Michel parameters.

CLEO-c [43] will run near tau threshold, which will allow it to measure the mass of the tau, and the tau neutrino, with greater precision. It will also be able to measure the Michel parameters using the unique spin correlations at tau threshold.

High statistics will allow rare decays to be seen and their Lorentz structure studied; e.g., the isospin violating mode $\tau \rightarrow \eta \pi \nu$, and the Wess-Zumino coupling in $\eta \pi \pi$.

High statistics will yield more stringent limits on (and perhaps an observation of) neutrinoless decays, anomalous (eg, CP violating) couplings in weak decay or in QED production, or exotica such as $\tau^{-} \rightarrow \pi^{-} v_{\text {heavy }}, e^{-} G^{0}$.

Taus will be used increasingly as a tool to study electroweak physics at the Tevatron and LHC, e.g., in Higgs decays $H^{0} \rightarrow \tau^{+} \tau^{-}, H^{-} \rightarrow \tau^{-} \bar{v}_{\tau}$.

Taus will continue to be a powerful probe of electroweak physics, tests of lepton universality, low energy meson dynamics, beyond-SM effects, and more. The B-Factories and CLEO-c will probe tau physics with ever greater precision and sensitivity. The study of tau lepton production and decay is still a very rich and active field.

\section{ACKNOWLEDGMENTS}

The author would like to thank all the physicists who have contributed to the explorations of tau physics that are surveyed here. Thanks also go to the organizers of this conference, who provided a pleasant environment and very stimulating program.

\section{REFERENCES}

1. TAU 2000, Proceedings of the Sixth International Workshop of Tau Lepton Physics, Victoria, British Columbia, Canada, September 2000. Published in Nucl. Phys. B (Proc. Suppl.) 98, (2001).

2. DELPHI Collaboration (P. Abreu et al.), Eur. Phys. J. C16, 229 (2000).

3. ALEPH Collaboration (A. Heister et al.), CERN EP/2001-035 (Submitted to Eur. Phys. J. C, 2001).

4. L3 Collaboration (P. Achard et al.), Phys. Lett. B519, 189 (2001).

5. DELPHI Collaboration (P. Abreu et al.), Eur. Phys. J. C20, 617 (2001).

6. M. Davier and A. Höcker, Phys. Lett. B435, 427 (1998); R. Alemany, M. Davier and A. Höcker, Eur. Phys. J. C12, 123 (1998); M. Davier and A. Höcker, Phys. Lett. B419, 419 (1998).

7. CLEO Collaboration (D. Asner et al.), Phys. Rev. D62, 072006 (2000).

8. CLEO Collaboration (A. Anastassov et al.), Phys. Rev. Lett. 86, 4467 (2001).

9. CLEO Collaboration (P. Avery et al.), Phys. Rev. D64, 092005 (2001).

10. CLEO Collaboration (G. Bonvicini et al.), CLNS 01/1766 (2001), (submitted to Phys. Rev. Lett., 2001).

11. Belle Collaboration (K. Abe et al.), BELLE-CONF-0118 (2001), obtainable at http://belle.kek.jp/conferences/LP01-EPS/ .

12. Belle Collaboration (K. Abe et al.), BELLE-CONF-0120 (2001).

13. Belle Collaboration (K. Abe et al.), BELLE-CONF-0019(2000).

14. Belle Collaboration (K. Abe et al.), BELLE-CONF-0119 (2001). 
15. Particle Data Group (D.E. Groom et al.), Eur. Phys. J. C15, 1 (2000), and 2001 off-year partial update for the 2002 edition available on the PDG WWW pages (URL: http://pdg.lbl.gov/).

16. ALEPH Collaboration (B. Barate et al.), Z. Phys. C76, 15 (1997).

17. ALEPH Collaboration (B. Barate et al.), Eur. Phys. J. C4, 409 (1998).

18. OPAL Collaboration (K. Ackerstaff et al.), Eur. Phys. J. C7, 571 (1999).

19. E. Braaten, S. Narison, and A. Pich, Nucl. Phys. B373, 581 (1992); F. Le Diberdier and A. Pich, Phys. Lett. B289, 165 (1992) and B286, 147 (1992).

20. C. Becchi, S. Narison, E. de Rafael, F.J. Yndurain, Z. Phys. C8, 335 (1981); D.J. Broadhurst, Phys. Lett. B101, 423 (1981); S.C. Generalis, J. Phys. G15, L225 (1989).

21. Particle Data Group (D.E. Groom et al.), Eur. Phys.J. C15, 1 (2000), pages 85-94, and references therein.

22. ALEPH Collaboration (R. Barate et al.), Eur. Phys. J. C11, 599 (1999); S. Chen, M. Davier, E. Gamiz, A. Höcker, A. Pich, J. Prades, Eur. Phys. J. C22, 31 (2001).

23. CLEO Collaboration (K. W. Edwards et al.), Phys. Rev. D61, 072003 (2000).

24. CLEO Collaboration (J. Bartelt et al.), Phys. Rev. Lett. 76, 4119 (1996); ALEPH Collaboration (D. Buskulik et al.), Z. Phys. C74, 263 (1997); CLEO Collaboration (M. Bishai et al.), Phys. Rev. Lett. 82, 281 (1999).

25. ALEPH Collaboration (D. Buskilic et al.), Z. Phys. C74, 263 (1997); CLEO Collaboration (M. Artuso et al.), Phys. Rev. Lett. 69, 3278 (1992).

26. CLEO Collaboration (A. Anastassov et al.), Phys. Rev. Lett. 86, 4467 (2001).

27. OPAL Collaboration (G. Abbiendi et al.), Eur. Phys.J. C13, 197 (2000); CLEO Collaboration (S. Richichi et al.), Phys. Rev. D60, 112002 (1999); ALEPH Collaboration (R. Barate et al.), Eur. Phys. J. C1, 65 (1998).

28. See, for example, submissions by A. Weinstein, G. Chen, and J. van Eldik, all in Proceedings of the Sixth International Workshop of Tau Lepton Physics, Victoria, British Columbia, Canada, September 2000, Published in Nucl. Phys. B (Proc. Suppl.) 98, (2001).

29. CLEO Collaboration (T. Bergfeld et al.), Phys. Rev. Lett. 79, 2406 (1997).

30. CLEO Collaboration (M.S. Alam et al.), Phys. Rev. Lett. 76, 2637 (1996).

31. ALEPH Collaboration (R. Barate et al.), Eur. Phys. J. C1, 65 (1998).

32. CLEO Collaboration (K. Edwards et al.), Phys. Rev. D56, 5297 (1997).

33. CLEO Collaboration (R. Godang et al.), Phys. Rev. D59, 091303 (1999); CLEO Collaboration (D. Bliss et al.), Phys. Rev. D57, 5903 (1998); CLEO Collaboration (G. Bonvicini et al.), Phys. Rev. Lett. 79, 1221 (1997); CLEO Collaboration (K. Edwards et al.), Phys. Rev. D55, 3919 (1997).

34. ARGUS Collaboration (H. Albrecht et al.), Z. Phys. C68, 25 (1995); ARGUS Collaboration (H. Albrecht et al.), Z. Phys. C55, 179 (1992).

35. MEGA Collaboration (M.L. Brooks et al.), Phys. Rev. Lett. 83, 1521 (1999).

36. CLEO Collaboration (S. Ahmed et al.), Phys. Rev. D61, 071101 (2000).

37. W. Bernreuther, U. Löw, J.P. Ma, O. Nachtmann, Z. Phys. C43, 117 (1989).

38. W. Bernreuther, O. Nachtmann, Phys. Rev. Lett. 63, 2787 (1989); W. Bernreuther, G.W. Botz, O. Nachtmann, P. Overmann, Z. Phys. C52, 567 (1991).

39. A. Zalite, in TAU '98, Proceedings of the Fifth International Workshop of Tau Lepton Physics, Santander, Spain, September 1998. Published in Nucl. Phys. B (Proc. Suppl.) 76, (1999).

40. ARGUS Collaboration (H. Albrecht et al.), Phys. Lett. B485, 37 (2000).

41. L3 Collaboration (M. Acciari et al.), Phys. Lett. B434, 169 (1998).

42. G.A. Gonzalez-Sprinberg et al., Nucl. Phys. B582, 3 (2000).

43. CLEO-c Collaboration (R. Briere et al.), CLNS-01-1742 (2001), obtainable at http://www.lns.cornell.edu/public/CLEO/spoke/CLEOc/. 